\title{
Rede Social no Sistema de Saúde: um Estudo das Relações Interorganizacionais em Unidades de Serviços de HIV/AIDS
}

Social Network Analysis in the Health System: a Study of Interorganizacional Relationships in HIV/AIDS Units

Otávio Neves da Silva Bittencourt * Doutor em Engenharia de Produção pela UFRGS. Assessor de planejamento do Hospital de Clínicas, Porto Alegre/RS, Brasil.

Francisco José Kliemann Neto Doutor em Engenharia de Produção pela Institut National Polytechnique, França. Professor Adjunto da UFRGS, Porto Alegre/RS, Brasil. citar parte de artigos sem autorização prévia desde que seja identificada a fonte. 


\section{RESUMO}

A coordenação dos serviços de saúde, obtida pela integração e a cooperação entre as unidades de um sistema local, passa pela compreensão das relações interorganizacionais, a identificação de unidades centrais e da dinâmica de trocas, tanto de pacientes, como de informações e conhecimento. O objetivo deste artigo é demonstrar as contribuições da análise de rede social para o sistema de saúde, identificando padrões e estruturas na rede de serviços, por meio de um estudo de caso na prestação de serviços de HIV/AIDS na cidade de Porto Alegre - Brasil. Por meio de entrevistas com os responsáveis pelo atendimento e dados do município, foram pesquisados cinco tipos de relacionamento: envio e recebimento de pacientes, coordenação de caso, programas conjuntos e consultorias. Os resultados indicam uma fraca dinâmica na rede, principalmente nos relacionamentos que não envolvem troca de pacientes, sugerindo que, se há instrumentos estabelecidos, como a marcação de consultas, há maior dinâmica na rede; do contrário apenas observam-se iniciativas locais ou pessoais, que redundam em pouca ou nenhuma ação de coordenação dos serviços. As medidas da rede social também identificam o papel das unidades, a formação de subgrupos e estas informações podem apoiar o gestor local na formulação de políticas e programas para o aprimoramento da governança local.

Palavras-chave: análise de rede social; avaliação da integração; gestão de sistemas de saúde; prestação de serviços em HIV/AIDS.

\section{ABSTRACT}

The coordination of health services, obtained by integration and cooperation between units of a local system, is achieved by understanding interorganizational relationships, identifying central units and dynamics flow, such as patients, information and knowledge. This paper demonstrates the contributions of social network analysis to the health system, identifying patterns and structures in the network of services, through a case study of the HIV/AIDS healthcare units in the city of Porto Alegre - Brazil. Through interviews with those responsible for the care and data from the council, five types of relationships were studied: forwarding and receiving patients, coordination of cases, joint programs and consultancies. The results indicate a weak dynamic in this network, particularly in relationships that do not involve an exchange of patients, suggesting that if there are established instruments such as scheduled appointments, there is greater dynamic in the network, if not, the personal or local initiatives result in little or no action in terms of coordination between services. The social network measures also identify the role of units and the formation of subgroups, and this information could support the local health authority in the formulation of policies and programs for the improvement of local governance.

Key words: social network analysis; integration assessment; healthcare system management; HIV/AIDS healthcare services. 


\section{INTRODUÇÃO}

A assistência de saúde compreende uma complexa seqüência de transações entre pacientes, prestadores de serviços e outras organizações ligadas à saúde (Stiles, Mick, \& Wise, 2001). O paciente do Sistema Único de Saúde [SUS] no Brasil com HIV/AIDS, por exemplo, pode ser diagnosticado e ter o primeiro atendimento numa unidade especializada, ser encaminhado para outra unidade para tratar de complicações decorrentes da doença, realizar exames diagnósticos em outro local, ser encaminhado para uma unidade de nível secundário, com maiores recursos tecnológicos, e retornar à primeira unidade. Conforme o problema de saúde e a característica local do sistema, esta seqüência pode ser modificada e acrescentados serviços mais elaborados.

Ainda que façam parte de um mesmo sistema e existam mecanismos de referência e contrareferência de pacientes entre as unidades de saúde, cada uma costuma tratar o paciente com escassas trocas de informações ou coordenação do tratamento e limitadas ações para o estabelecimento de objetivos comuns de saúde ou organizacionais. Esta costuma ser a forma tradicional como as unidades de saúde prestam o serviço.

A nova visão trazida dos estudos de arranjos estruturais de sistemas de saúde altera o foco para o de coordenação dos serviços, através da governança entre as organizações. A governança, neste contexto, refere-se à habilidade, ao conhecimento e à prática de coordenação de múltiplas organizações para que o sistema funcione como um organismo coeso e focado em objetivos comuns. Cada ator organizacional tem vontade própria para agir dentro de seu papel, não sendo possível obrigá-lo a adotar as regras ditadas por outros integrantes da rede. Por outro lado, atingir um desempenho superior e ofertar melhores produtos e serviços em ambientes complexos, muitas vezes, não é tarefa para ser feita isoladamente. Decorre daí o principal desafio da governança interorganizacional também para a saúde.

A definição de políticas públicas e a gestão de sistemas de saúde requerem uma visão abrangente e ao mesmo tempo específica a respeito dos fenômenos de troca, seja de pacientes ou de informações. A implementação de uma política no sistema pode ser facilitada na medida em que houver conhecimento das unidades mais influentes. No exemplo acima, saber como estão estabelecidas as relações organizacionais entre as unidades que prestam serviços a pacientes com HIV/AIDS e quais são as unidades centrais e de maior influência podem auxiliar a tomada de decisão em sistemas de saúde.

Para responder a estes questionamentos optou-se por abordar este problema à luz da análise de rede social, um ramo do campo de estudo das relações interorganizacionais, capaz de monitorar a dinâmica de trocas presentes na produção do serviço de saúde.

O objetivo deste artigo é demonstrar as contribuições da análise de rede social para o sistema de saúde, através de medidas de interação que demonstram a dinâmica e a estrutura de uma rede de serviços.

Para evidenciar essa proposta, é apresentado um estudo de caso que analisa as unidades de saúde prestadoras de serviços a pacientes vivendo com HIV/AIDS na cidade de Porto Alegre (RS), Brasil. A partir da rede de serviços definida pela Secretaria de Saúde do município, foram pesquisados cinco tipos de relacionamento, também presentes em outros estudos (Kwait, Valente, \& Celentano, 2001; Provan \& Milward, 1995): referência de envio, referência de recebimento, coordenação de caso, programas conjuntos e consultorias. Para a identificação de cada tipo de relacionamento, foram realizadas entrevistas com os responsáveis pelo atendimento aos pacientes das unidades de saúde e utilizados os dados disponíveis do sistema de informações de marcação de consultas do município.

Este artigo está estruturado em sete seções que buscam inicialmente apresentar o referencial teórico e situar o leitor na problemática proposta, para a seguir apresentar o estudo de caso. Além desta introdução, a segunda seção posiciona a rede social no marco teórico das relações interorganizacionais e apresenta as principais contribuições da análise de redes sociais ao campo organizacional, além dos 
conceitos a serem estudados. A terceira seção apresenta a forma de organização do sistema de saúde para pacientes com HIV/AIDS no Brasil e contextualiza este sistema no município de Porto AlegreRS, unidade de análise desta pesquisa. A quarta seção apresenta os procedimentos metodológicos e os fatores de análise empregados. A quinta seção discorre sobre os resultados do estudo de caso. $\mathrm{O}$ artigo encerra com as considerações finais sobre as implicações dos achados para a prática da gestão de sistemas de saúde.

\section{RELAÇÕES INTERORGANIZACIONAIS E REDES}

O estudo de Oliver e Ebers (1998) identificou duas vertentes no campo de estudo das relações interorganizacionais e redes: a rede social e a governança. A mesma conclusão é assinalada por Martes, Loureiro, Abramovay, Serva e Serafim (2006). A primeira vertente é subdividida em: a) rede social; b) poder e controle. A segunda subdivide-se em: a) teoria institucional; b) economia institucional e estratégia.

A vertente Rede Social emprega o aparato formal da análise de rede social com vistas a examinar como as propriedades estruturais das redes internas e externas e as posições dentro de tais redes influenciam as organizações e seus membros (Oliver \& Ebers, 1998, p. 568).

Por outro lado, a vertente Governança abarca todas as abordagens teóricas que estudam os mecanismos institucionais pelos quais os relacionamentos interorganizacionais são iniciados, negociados, desenhados, coordenados, monitorados, adaptados e terminados. Em oposição à Rede Social, esta perspectiva focaliza menos as propriedades estruturais dos relacionamentos interorganizacionais e as posições dos atores, pelo contrário, concentra-se nos atributos tanto de atores como da forma e conteúdo dos relacionamentos, dentro de um contexto institucional em particular (Oliver \& Ebers, 1998, p. 569).

As pesquisas que se baseiam na análise de redes sociais preocupam-se em examinar como diferentes posições, dentro de uma teia de relacionamentos, afetam as oportunidades dos detentores de tais posições (Oliver \& Ebers, 1998, p. 568); examinam também a emergência e a mudança de estruturas informais, os limites das redes, o processo de cooptação corporativa, as interconexões de diretorias e os padrões de relações entre pequenas empresas, vinculando-se todos esses aspectos ao campo da Psicologia Social (Grandori \& Soda, 1995, pp. 191-192).

A perspectiva de Poder e Controle estuda as características estruturais das redes, identificando funções e organizações vinculadas a elas, bem como o controle interorganizacional (Oliver \& Ebers, 1998, p. 569). Ela está vinculada aos estudos organizacionais, em especial da administração pública (Grandori \& Soda, 1995, pp. 186-187).

Não resta dúvida quanto às contribuições de ambas as vertentes para o sistema de saúde; porém optou-se pela rede social, porque se entendeu que ela pode responder às indagações formuladas anteriormente, por se tratar de ambiente organizacional público, pelo potencial de contribuição ao setor de saúde, como demonstra estudo anterior (Kwait et al., 2001) e pela viabilidade de operacionalização na rede local de saúde.

\section{Análise de Redes Sociais no Campo das Organizações}

A Análise de Redes Sociais, denominada também de Sociologia Relacional, é o estudo dos relacionamentos que envolvem as conexões de atores para auxiliar no esclarecimento dos fenômenos sociais (Marques, 2000, p. 33). Estas análises observam de que forma a localização na rede se relaciona com poder e influência, através do fluxo de trocas entre os atores. 
As raízes da análise de redes sociais são bem diversas e antigas. No século XVIII há registros de uma aplicação matemática para resolver o problema das pontes da cidade de Königsberg (Luke \& Harris, 2007). Cientistas sociais, psicólogos educacionais, antropólogos, psiquiatras e sociólogos contribuíram para erguer esta disciplina (Freeman, 1996; Luke \& Harris, 2007; Mizruchi, 1994) que alcança o auge de seu crescimento na segunda metade do século XX, acompanhando a transformação das abordagens individualista, essencialista e atomística para uma compreensão mais relacional, contextual e sistêmica dos fenômenos (Borgatti \& Foster, 2003).

Os efeitos desta disciplina na teoria organizacional começaram a ser observados, quando os modelos de racionalidade, baseados nos pressupostos de uma organização individual, passaram a ser pautados pelos relacionamentos interorganizacionais. Nesta abordagem, a organização passa a ser um nó ou ator num conjunto de relacionamentos sociais (Milward \& Provan, 1998). Cada conteúdo dos laços de uma rede (como informação ou amizade) define uma rede distinta com funções e estruturas específicas. Uma rede de ações de cooperação, muito provavelmente, terá uma forma diferente de uma rede de conflitos (Borgatti \& Foster, 2003).

Até a metade da década de 80 , havia poucas evidências de que a teoria da análise de redes sociais pudesse contribuir para o estudo das relações interorganizacionais. A partir daí houve uma proliferação de estudos sugerindo que o posicionamento numa rede influencia o comportamento da organização em face das demais. Muitos destes estudos voltaram-se para as relações entre empresas a partir dos vínculos dos integrantes dos conselhos de administração e diretorias. Esses estudos concluíram, por exemplo, que tais vínculos têm um impacto significativo nas estratégias corporativas (Mizruchi, 1994).

Em termos gráficos, todas as formas de análise sociométricas especificam que os pontos ou nós de um gráfico são atores (pessoas ou organizações) e que as linhas ou laços dos gráficos são relacionamentos sociais, em que atores e relacionamentos são concebidos como fenômenos irredutíveis (Breiger, 1974).

Numa análise de rede, em geral, observam-se aspectos como densidade média, cliques, transitividade, centralidade e centralidade de intermediação (Grandori \& Soda, 2000; Marques, 2000).

A densidade média está relacionada com a consistência interna de uma rede, com a capacidade de oferecer suporte social e bem-estar e com o nível de confiança entre os seus integrantes (Kadushin, 2002; Marsden, 1987). O cálculo realiza-se considerando o número de vínculos sobre o total possível, onde o total de vínculos possíveis é obtido através do cálculo de análise combinatória, pela combinação de $n$ integrantes da rede, dois a dois (Marques, 2000, p. 223). Quanto maior a densidade, em tese, mais força potencial a rede tem para oferecer uma resposta (Marsden, 1987).

O cliques (ou grupo coexo) é um subgrupo de uma rede completamente conectado, ou seja, todos os nós estão conectados a todos os outros (Grandori \& Soda, 2000, p. 8). O cliques representa a formação de estruturas dentro de uma rede (Hanneman \& Riddle, 2005), uma vez que raramente há uma uniformidade de conexões quando, na realidade, alguns integrantes se conectam mais do que outros e há uma preferência por conectar-se com um e não com outros. Quanto maior a densidade de uma rede, menor a chance de haver cliques numa rede (Hanneman \& Riddle, 2005, p. 78).

A transitividade representa o número de conjuntos de três nós que atualmente estão conectados (triplos ordenados), sobre o número total de triplos potencialmente conectados. Em particular, a transitividade permite avaliar se as interdependências são predominantemente lineares (sugerindo que a rede tem formato de cadeia), ou é rica de círculos ou recirculações (sugerindo que a rede é uma teia). A regra geral da transitividade afirma que os atores $i, j$ e $k$ são transitivos se $i->j, j->k$, então $i->k$, de forma que o fluxo das conexões se apresenta sem antagonismos (Wasserman \& Faust, 1994, pp. 243245). A transitividade favorece a formação de cliques num processo de escolhas de novas interações a partir das conexões já conhecidas pelos atores agora conectados (Feld \& Elmore, 1982).

A centralidade de um nó se define pela quantidade de conexões de um ator ou deste a outros atores com grande número de conexões. Nestas situações, usualmente, o ator central tem relativamente alto 
impacto sobre os resultados e pode ser candidato ao papel de coordenação (Marques, 2000, p. 224). A centralidade pode estar vinculada com a capacidade de o ator influenciar os demais em razão do maior domínio da informação (Mizruchi, 1994). Em algumas situações, os atores podem preferir colaborar com um determinado integrante pela riqueza na troca de informações e conhecimentos, resultando em redes com menor densidade (Rank, Rank, \& Wald, 2006).

A centralidade de intermediação (betweeness) representa indivíduos, grupos ou organizações que são o único caminho para outro grupo e, em tais condições, detêm grande poder sobre o restante do grupo e podem também contar com poder e prestígio junto ao restante da rede, visto que sua ausência provocaria uma ruptura na rede de relações (Marques, 2000, p. 225). Esse índice é obtido pelo somatório das probabilidades de um ator estar no caminho de outros dois atores da rede (chamado caminho geodésico). Assim, por exemplo, os atores j e k comunicam-se por meio de gjk caminhos; o ator i está no caminho desses dois atores em gjk(ni), assim gjk(ni)/gjk representa a probabilidade do ator $\mathrm{i}$ estar entre os caminhos de $\mathrm{j}$ e $\mathrm{k}$. Esse cálculo é efetuado de um ator da rede para todos os demais, onde se obtém o somatório de probabilidades do ator i (Wasserman \& Faust, 1994, pp. 189191). Um ator nesta posição pode comportar-se negativamente, interrompendo e distorcendo o fluxo da rede, ou positivamente, servindo de incentivador de atores distantes do núcleo central (Owen-Smith \& Powell, 2004, p. 16).

\section{Poder e Controle em Redes Sociais}

A literatura indica que há uma relação direta entre o tamanho da rede e a necessidade de coordenação para uma efetiva integração entre os diversos elementos (Provan \& Milward, 1995). Um dos grandes desafios da organização em rede reside em estabelecer modalidades de gerência que permitam alcançar os objetivos previstos e manter sua organicidade. A compreensão dos fenômenos de poder e controle podem apoiar-se na formulação de estratégias para a rede que alcancem esta finalidade.

Estes conceitos podem ser verificados pela aplicação do método reputacional, complementando a leitura anterior, por exemplo indagando aos membros de uma rede qual o ator mais influente (Tichy, Tushman, \& Fombrun, 1979). Com esta avaliação em mãos, Provan e Milward (1995) propõem uma análise da integração da rede por meio de medidas complementares: a densidade e a centralização.

A densidade da rede pode ser calculada pela multiplexidade de serviço que é a média do escore de ligações dos atores do serviço, obtido pelo número total de conexões dos atores da rede dividido pela soma dos escores previstos de todos os tipos de ligação para os atores da rede. Essa medida reflete a profundidade, força ou durabilidade da integração da rede. Escores moderadamente altos podem ser atingidos por relativamente poucos atores integrados a todos os demais ou a sua maior parte.

As medidas de centralização compreendem o nível de centralidade do ator principal, com os escores de densidade apenas para a agência principal, e a concentração da influência, medida pela taxa de ligação da rede com o ator mais influente.

A operacionalização dessas medidas é feita através da criação de escores que identificam o ator mais influente da rede e examinam a distribuição da influência, focando na distância entre os escores do ator mais influente e aquele ou aqueles na segunda posição. Se o segundo ator não tem mais do que a metade do escore de influência do ator top, então se considera que o sistema tem a influência concentrada (Provan \& Milward, 1995, p. 15).

O estudo das redes sociais parte das ligações entre organizações, seja num momento específico da rede ou através de um estudo que compreende um período de tempo, para daí derivar sua análise das relações entre as organizações e da situação do controle e poder na rede. 


\section{ESTUDO DAS REDES SOCIAIS EM SAÚDE}

O problema da integração nos sistemas de saúde tem sido abordado como desafio presente e necessário de uma forma geral (Conrad \& Shortell, 1996; Friedman \& Goes, 2001; Institute of Medicine, 2001; Light, 2001). No sistema brasileiro e em outros países com poucos recursos, esse problema talvez seja ainda maior por fatores como a origem e a diversidade das organizações, e a superioridade das demandas diante da capacidade de resolução, principalmente para o setor público.

A tendência demográfica e a própria medicina estão mudando o perfil epidemiológico para doenças crônicas. Doenças crônicas requerem uma forma diferente de organização da saúde, se comparada com situações agudas (The Institute for the Future, 2003, p. 79). A ênfase está no cuidado, ao invés da cura, no monitoramento, ao invés da intervenção aguda, na multidisciplinaridade e na criação de redes de saúde integradas (Delnoij, Klazinga, \& Velden, 2003). A integração e a coordenação de serviços, como no caso da assistência em HIV, podem otimizar o uso dos recursos e aumentar o acesso (Kitahata, Tegger, Wagner, \& Holmes, 2002).

No Brasil, por exemplo, estudos das redes em saúde, formadas pelos diversos sistemas locais do Sistema Único de Saúde [SUS], apontam alguns problemas como: a) distorções e ineficiências do investimento, acarretando a implantação incompleta de redes intermediárias e básicas de serviços, o que sobrecarrega e compromete a efetividade do sistema hospitalar; b) a ineficiência, mas principalmente a ineficácia dos recursos; c) modelo assistencial fundado na livre demanda, do qual estão ausentes os princípios de adscrição de clientela e de identificação de porta de entrada; d) organização e gestão antiquadas e inadequadas do sistema e de suas unidades organizacionais (Draibe, 1999, pp. 129-130). Os municípios, responsáveis por gerir estes sistemas de saúde, têm uma rede de serviços heterogênea e não integrada (Monnerat, Senna, \& Souza, 2002).

As longas filas de espera, a demora no atendimento e as dificuldades de obter atendimento sugerem que a assistência não está desenhada para o bem-estar do paciente, ou seja, não está centrada nas necessidades do cliente (Institute of Medicine, 2001).

A rede de serviços de saúde integrada ainda é um dos melhores mecanismos disponíveis para prestar assistência às comunidades com alta qualidade e de maneira custo-efetiva. $\mathrm{O}$ valor central da integração é aperfeiçoar tanto a coordenação e a integração de diferentes atores do sistema de saúde, sejam eles da própria comunidade ou de unidades de referência, como a experiência dos pacientes e os resultados de saúde (Friedman \& Goes, 2001).

Levando-se em conta o ambiente do sistema de saúde, esta integração muito provavelmente não será fácil, razão pela qual é colocada como desafio. O estudo das redes sociais para a saúde proporciona informações que podem facilitar a gestão da rede e facilitar seu alcance. Para Moe (2005, p. 280), a gestão de uma rede envolve ações de desenvolvimento e apoio às relações interpessoais ou informais, a fim de obter resultados efetivos das unidades participantes da rede, num processo longo e contínuo. $\mathrm{O}$ estudo das redes sociais em saúde fornece ao gestor do sistema os elementos para o desempenho desta atividade.

\section{A Organização da Prestação de Serviços de Saúde para pacientes com HIV/AIDS}

O sistema de saúde brasileiro está organizado em sistema público, SUS e sistema complementar. No primeiro, o pagador é o Estado, e no segundo são os operadores de planos de saúde, tanto entidades públicas como privadas. No SUS, as esferas de Governo Federal, Estadual e Municipal participam da definição de políticas, execução e aporte de recursos dentro do âmbito de responsabilidade que a legislação estabelece. 
O atendimento a pacientes com HIV/AIDS é realizado, em sua maioria, pelo sistema público, que oferece atenção em saúde integral a esse tipo de doença através de um programa de saúde de referência mundial. Neste caso, o Ministério da Saúde tem papel preponderante na política nacional, definindo os parâmetros de tratamento, planejando o desenvolvimento de fármacos, medicamentos e de testes diagnósticos, criando uma estrutura de monitoramento da resposta dos pacientes à medicação e participando da execução do programa nacional, por exemplo na aquisição de medicamentos, na gestão do sistema de monitoramento de indicadores e do sistema de controle logístico de medicamentos anti-retrovirais.

O atendimento ao paciente é de responsabilidade do município, onde o sistema está organizado em unidades especializadas, para oferecer o acolhimento, orientação, consulta, exames diagnósticos, tratamento com medicamentos, apoio a organizações não-governamentais e ações de prevenção. Esta rede de unidades especializadas se integra às demais unidades de saúde do sistema público, para o atendimento integral em saúde.

A unidade de análise desta pesquisa é a rede de serviços para HIV/AIDS do município de Porto Alegre. A cidade de Porto Alegre tem 1.453.076 habitantes, representando 13,11\% da população do Estado do Rio Grande do Sul, com um nível de alfabetização de 94,3\% e uma taxa de crescimento anual da população estimada em $0,8 \%$. Como ocorre em muitas capitais brasileiras, a cidade concentra alguns recursos de saúde do Estado, como 46,58\% dos procedimentos ambulatoriais de alta complexidade, $36,68 \%$ do valor pago com internações e $23,67 \%$ dos pagamentos e transferências efetuadas aos prestadores de serviços do SUS. Considerando todos os estabelecimentos de saúde do Estado, Porto Alegre participa com 15,14\%, próximo da participação populacional, o que revela sua função de referência em saúde no sistema estadual para a realização de atendimentos mais complexos. O município de Porto Alegre foi habilitado pelo Ministério da Saúde para gestão plena, assumindo a responsabilidade pela gestão do sistema de saúde municipal (Ministério da Saúde [MS], 2007).

Em 2005, as famílias de Porto Alegre gastaram 5,4\% do rendimento familiar com saúde. A cobertura de planos privados de saúde de Porto Alegre foi de 35,58\%. Neste ano, a taxa de incidência de AIDS em Porto Alegre foi de 61,59 casos por 100.000 habitantes (Ministério da Saúde, 2007).

O total acumulado de casos de AIDS em Porto Alegre, de 1983 a 2006, é de 15.301 casos, com coeficiente de prevalência de 587,20 casos por 100.000 habitantes. O diagnóstico tardio do HIV e/ou AIDS e a falha na adesão aos anti-retrovirais são causas prováveis da letalidade de $21,7 \%$ no ano de 2006 (Secretaria Municipal de Saúde de Porto Alegre, 2007). A doença está entre as 8 principais causas de óbito, com 36,54 por 100.000 habitantes (Ministério da Saúde, 2007).

\section{Procedimentos MetOdOLÓGICOS}

Em razão do tipo de problema e dos objetivos da pesquisa, bem como dos referenciais bibliográficos, optou-se por um estudo de caso com a aplicação de questionário ao responsável técnico das unidades de saúde especializadas em HIV de Porto Alegre (Tabela 1). Nesta entrevista foram identificados quais dos cinco tipos de relacionamento (referência de envio, referência de recebimento, coordenação de caso, programas conjuntos e consultorias) estavam presentes no atendimento a estes pacientes, e também qual a influência das mesmas unidades nas decisões internas em relação ao atendimento dos pacientes com HIV/AIDS, por ordem de prioridade. As entrevistas foram realizadas de outubro de 2004 a janeiro de 2005.

Todas as 16 unidades foram contatadas; em duas unidades o entrevistado coordenava o Serviço de Assistência Especializada [SAE] e os Centros de Testagem e Aconselhamento [CTA] (SAE e CTA Murialdo; SAE e CTA Ambulatório de Dermatologia Sanitária). Outras duas unidades, por questão de disponibilidade, não responderam (Irmandade Santa Casa de Misericórdia e Hospital da Criança Santo Antônio) e o ADOT estava praticamente desativado, resultando em 11 entrevistados. Destes, apenas 5 
acharam-se em condições de responder à questão de influência nas decisões internas, e os demais entenderam que isto não ocorre.

Tabela 1: Unidades Especializadas em HIV/AIDS de Porto Alegre

\begin{tabular}{|l|l|}
\hline Unidade de Saúde & Vinculação \\
\hline Serviço de Assistência Especializada [SAE] - Murialdo/Partenon & Estadual \\
\hline Serviço de Assistência Especializada [SAE] da Secretaria Municipal de Saúde & Municipal \\
\hline Ambulatório de Dermatologia Sanitária (SAE) & Estadual \\
\hline Hospital Nossa Senhora da Conceição - GHC & Federal \\
\hline Hospital Fêmina - GHC & Federal \\
\hline Hospital de Clínicas de Porto Alegre & Federal \\
\hline Hospital Vila Nova & Privado \\
\hline Irmandade Santa Casa de Misericórdia & Privado \\
\hline Hospital São Lucas - PUC/RS & Privado \\
\hline Hospital Sanatório Partenon & Estadual \\
\hline Hospital da Criança Santo Antônio & Privado \\
\hline Hospital Materno Infantil Presidente Vargas & Municipal \\
\hline CTA/COAS Paulo César Bonfim & Municipal \\
\hline CTA/COAS do Ambulatório de Dermatologia Sanitária & Estadual \\
\hline CTA/COAS Murialdo/Partenon & Estadual \\
\hline Assistência Domiciliar Terapêutica a Portadores de AIDS (ADOT) & Municipal \\
\hline
\end{tabular}

A Secretaria de Saúde do município possui o registro das consultas marcadas pelas unidades de saúde da rede para as unidades especializadas (referência de recebimento). A referência de envio não foi possível resgatar do sistema de informações, tendo sido obtida pelo relato dos entrevistados. Sabese que essa forma de coleta de dados apresenta deficiências pela dificuldade do entrevistado em relembrar inúmeros eventos (Fontana \& Frey, 2000, p. 650), sendo esta uma das limitações do estudo. Para os demais relacionamentos, em menor número, acredita-se que o relato, durante a entrevista, se aproxime da realidade. O relacionamento por intermédio de consultorias não foi observado na rede.

A análise das relações organizacionais dessa rede compreende a identificação das unidades mais conectadas, as centrais e intermediárias, a formação de subgrupos e as interconexões entre as unidades. Em função disso, as categorias de análise adotadas para tratar dos dados de cada tipo de relacionamento são a densidade média, cliques, transitividade, centralidade de um nó e posição de intermediário da rede, atributos definidos pela literatura. As medidas de cliques, transitividade, centralidade de um nó e posição de intermediário, bem como a representação gráfica do sociograma são elaborados com o apoio do UCINET versão 6 (Borgatti, Everett, \& Freeman, 2002). Na medida de cliques foi utilizado o número mínimo de 3 integrantes por subgrupo.

Para o estudo do poder e controle, as categorias de análise foram multiplexidade de serviço, nível de centralidade do ator principal e concentração da influência.

\section{ANÁLISE DAS RELAÇÕES ENTRE ORGANIZAÇÕES DA REDE DE HIV/AIDS}

A estrutura de atendimento para HIV/AIDS é formada pelos Centros de Testagem e Aconselhamento [CTA], também denominados de Centro de Orientação e Apoio Sorológico [COAS], e pelos Serviços de Assistência Especializada [SAE]. O SAE é responsável pelo acompanhamento do paciente, em razão da condição crônica da doença, e pela distribuição dos medicamentos. Como a doença enseja o surgimento de outras complicações, o paciente precisa buscar os serviços de saúde não especializados para o tratamento destes episódios. 
Os CTAs funcionam como locais de acolhimento de pessoas que estejam com suspeitas de estarem infectadas. Para ampliar o acesso ao diagnóstico precoce da infecção pelo HIV e ao aconselhamento, dentro de normas e princípios que não firam os direitos humanos e garantam a voluntariedade na realização da sorologia anti-HIV, a coordenação nacional de Doenças Sexualmente Transmissíveis [DST] e AIDS vem promovendo, em conjunto com Estados, Municípios e Universidades, a implantação desses Centros. Trata-se de unidades de saúde que oferecem o diagnóstico sorológico da infecção pelo HIV, de forma gratuita, atendendo à demanda, tanto espontânea quanto provocada.

A coordenação de DST do município de Porto Alegre considera como serviços especializados as unidades públicas, ligadas ao Município, ao Estado e à União, assim como as unidades privadas, todas vinculadas ao SUS, conforme apresentado na Tabela 1. Os três SAEs (Murialdo, SAE Municipal e o Ambulatório de Dermatologia Sanitária) possuem CTAs vinculados a eles, o que lhes confere, a princípio, uma posição privilegiada na estrutura de serviços como porta de entrada no sistema. Esse tema será objeto de análise adiante.

Os hospitais funcionam como serviço especializado em nível ambulatorial, como uma unidade de saúde para atendimentos de complicações decorrentes da doença, além de realizarem testes de forma anônima.

Os resultados da coleta de dados, para cada relacionamento, são apresentados na Tabela 2. No relacionamento de envio, o paciente é, geralmente, encaminhado para tratamento perto de sua residência. No relacionamento de recebimento, a Central de Marcação oferece as consultas conforme a disponibilidade da unidade especializada. Estes dois tipos de relacionamento funcionam por meio de mecanismos já estabelecidos na rede, enquanto os demais relacionamentos são iniciativas locais ou pessoais de cada unidade especializada.

A interpretação da densidade se dá pelo contexto da rede e pelo número de atores (Rank et al., 2006, p. 78). Neste caso, chama a atenção que uma rede voltada a atender uma doença crônica como o HIV/AIDS, que requer um longo e contínuo tratamento, tenha relacionamentos do tipo coordenação de caso e programas conjuntos, expressões de cooperação em saúde, pouco densas.

Tabela 2: Resumo da Análise entre Organizações

\begin{tabular}{|c|c|c|c|c|}
\hline Fator & Envio & Recebimento & Coordenação de Caso & $\begin{array}{c}\text { Programas } \\
\text { Conjuntos }\end{array}$ \\
\hline Densidade & $14 \%$ & $27 \%$ & $7,5 \%$ & $4,5 \%$ \\
\hline cliques & $\begin{array}{c}38(44,7 \%-\mathrm{SAE} \\
\text { Município e Hospital } \\
\text { Vila Nova) }\end{array}$ & $\begin{array}{c}133(36,1 \%- \\
\text { Hospital São Lucas e } \\
\text { Hospital N. Sra. } \\
\text { Conceição) }\end{array}$ & $\begin{array}{c}17(41,2 \% \text { - CTA } \\
\text { Partenon e SAE } \\
\text { Murialdo })\end{array}$ & $\begin{array}{c}10(80,0 \% \text { - CTA } \\
\text { Partenon e SAE } \\
\text { Murialdo })\end{array}$ \\
\hline Transitividade & $100 \%$ & $100 \%$ & $100 \%$ & $100 \%$ \\
\hline $\begin{array}{l}\text { Centralidade } \\
\left.\text { ( } \mathrm{n}^{\circ} \text { de conexões }\right)\end{array}$ & $\begin{array}{l}\text { Hospital Vila Nova } \\
\text { (71) } \\
\text { SAE Sec. Mun. de } \\
\text { Saúde (37) }\end{array}$ & $\begin{array}{l}\text { Hospital São Lucas } \\
\text { PUC (63) } \\
\text { Hosp. N. Sra. } \\
\text { Conceição (60) }\end{array}$ & $\begin{array}{l}\text { SAE Sec. Mun. Saúde } \\
(28) \\
\text { Amb. Derm. Sanitária } \\
\text { (16) } \\
\text { SAE Murialdo (16) }\end{array}$ & $\begin{array}{l}\text { Amb. Derm. } \\
\text { Sanitária (15) } \\
\text { Hosp. N. Sra. } \\
\text { Conceição (12) }\end{array}$ \\
\hline $\begin{array}{l}\text { Intermediação } \\
\text { (índice) }\end{array}$ & $\begin{array}{l}\text { Hospital Vila Nova } \\
(537,5) \\
\text { SAE Sec. Mun. } \\
\text { Saúde }(230,5)\end{array}$ & $\begin{array}{l}\text { Hospital São Lucas } \\
(207,5) \\
\text { Hosp. Mat. Inf. Pres. } \\
\text { Vargas }(55,5)\end{array}$ & $\begin{array}{l}\text { SAE Sec. Mun. Saúde } \\
(223) \\
\text { Hosp. Clínicas de P. } \\
\text { Alegre (96) } \\
\text { CTA/COAS Municipal } \\
\text { (82) }\end{array}$ & $\begin{array}{l}\text { CTA Partenon } \\
(96,5) \\
\text { Hospital N. Sra. } \\
\text { Conceição (84) }\end{array}$ \\
\hline
\end{tabular}

A inexistência de consultorias é outro aspecto que aponta a ainda incipiente dinâmica de trocas desta rede. A prática de consultorias requer disponibilidade de recursos financeiros e contínua colaboração 
(Kwait et al., 2001, p. 482), sendo, portanto, uma evidência de integração entre unidades, com efetiva troca de conhecimento e informações.

A análise de cliques apontou a presença de 38 nos relacionamentos de envio, 133 de recebimento, 17 de coordenação de caso e 10 de programas conjuntos. A grande maioria destes cliques contou com a participação de atores centrais, isto é, ao mesmo tempo que mantinham muitas conexões ainda estavam presentes na maioria dos subgrupos da rede. A exceção foi o relacionamento da coordenação de caso, onde $80 \%$ dos cliques tiveram a participação de outras unidades, sendo, neste caso, atores emergentes nesta rede (Hanneman \& Riddle, 2005).

Todos os relacionamentos atingiram $100 \%$ de transitividade, indicando não haver, em princípio, antagonismos entre os atores. Essa afirmação é apenas uma suposição teórica, que na verdade necessita ser confirmada por intermédio de outras medidas não previstas nesta pesquisa.

Os fatores finais, centralidade e intermediação, analisam as posições dos atores na rede. De maneira geral, os hospitais e o SAE da Secretaria Municipal de Saúde ocupam essas posições. Esse talvez seja um reflexo da antiga concepção do sistema de saúde, centrada na organização hospitalar, que se está tentando modificar com o SUS, para uma estruturação em rede com diferentes níveis de complexidade.

A identificação da centralidade nas redes sociais ganha relevância em função do papel exercido pela organização líder. A próxima seção se propõe a aprofundar essa análise, a fim de se verificar se há uma unidade central e qual é o grau de poder sobre a rede.

Nos relacionamentos de envio, a densidade média foi de $14 \%$, ao passo que no estudo de Kwait et al. (2001, p. 475) a densidade foi de 43,1\%. A centralidade e a intermediação são do Hospital Vila Nova e do SAE da Secretaria Municipal de Saúde. Este Hospital serve como retaguarda dos hospitais e unidades de saúde e, com isso, após o atendimento, envia o paciente às unidades de saúde mais próximas de sua residência. Este $\mathrm{SAE}$, por sua vez, é uma unidade de referência do município que faz o mesmo papel, embora com menor expressão na rede.

Nos relacionamentos de recebimento, as Unidades de Saúde prestam a assistência aos pacientes nas especialidades básicas, como clínico geral, ginecologia, pediatria e otorrinolaringologia. No caso da identificação ou suspeita de HIV/AIDS, o paciente é encaminhado ao serviço especializado, por intermédio da marcação de consultas da Secretaria da Saúde do município. A Secretaria possui agendas dos SAEs e hospitais nas especialidades de Infectologia Adulto e Pediátrica e é com base neste sistema que foram obtidos os dados desse relacionamento. No estudo de Kwait et al. (2001, p. 477) a densidade média foi de $33,3 \%$, em comparação com $27 \%$ para o presente estudo. Os atores mais centrais nessa rede são o Hospital São Lucas da PUC e o Hospital Nossa Senhora da Conceição. A Figura 1 apresenta essa rede de modo a demonstrar graficamente os relacionamentos.

A identificação dos atores centrais nesse relacionamento aponta uma aparente inconsistência com a política estabelecida para o funcionamento da rede. Os SAEs deveriam ser as principais unidades receptoras de pacientes do sistema, mas somente o SAE da Secretaria Municipal de Saúde figura entre essas unidades.

Detendo-se com maior atenção sobre esse fato, nota-se que os dois SAEs estaduais não disponibilizavam agendas de consulta para a Central de Marcação, configurando uma adesão parcial à política municipal. Ainda que a questão institucional não seja o enfoque dessa pesquisa, esse tema foi comentado pelo entrevistado da coordenação municipal e também pelos entrevistados dos SAEs estaduais. 


\section{Figura 1: Relacionamentos de Recebimento na Cadeia de Saúde de HIV/AIDS}

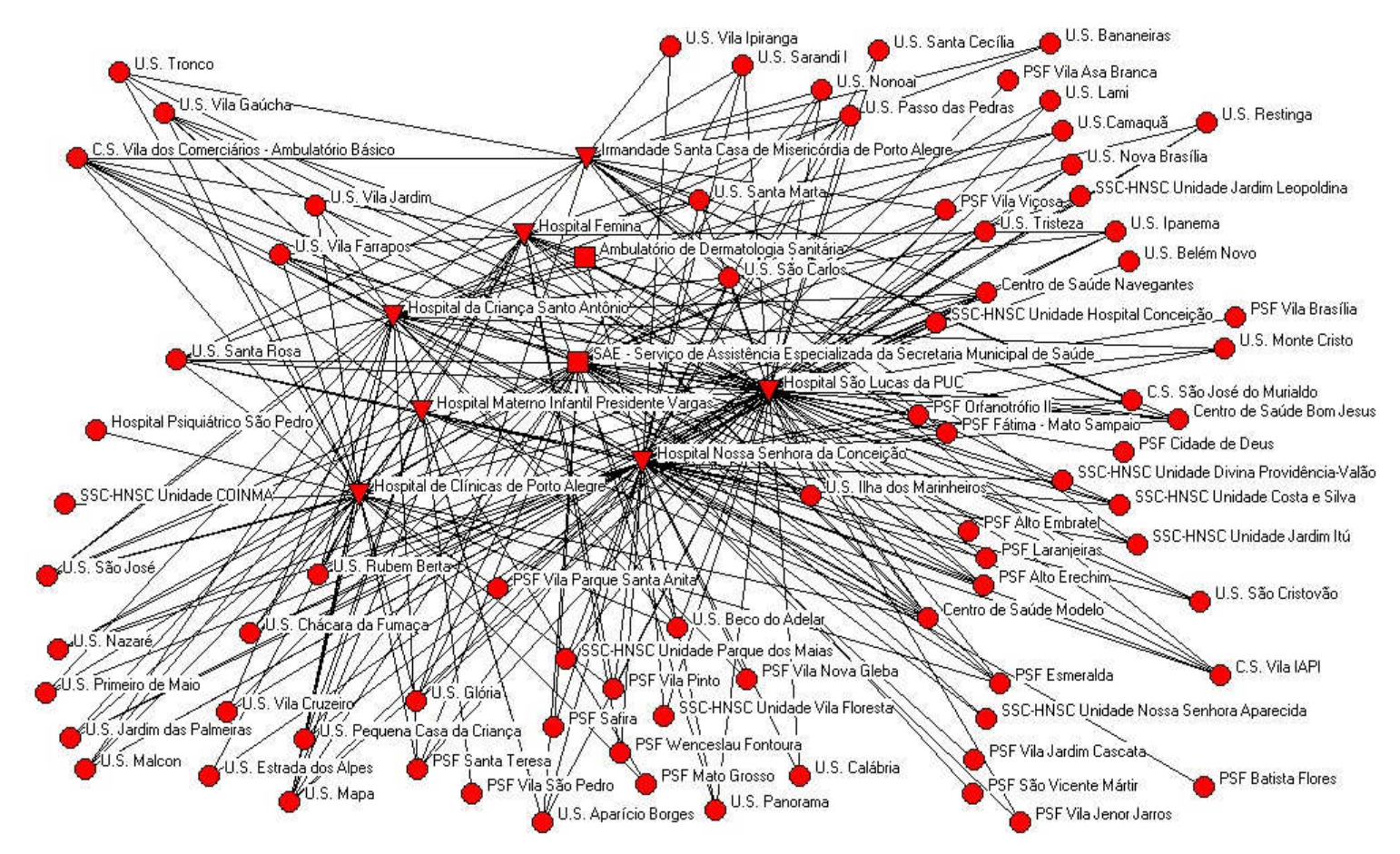

Legenda: $\square$ = SAE; $\nabla$ = Hospital com agenda de infectologia; $\bigcirc=$ outras unidades de saúde.

Parte do problema tem origem na estruturação do SUS quando da municipalização, ou seja, a delegação ao município da coordenação da saúde na cidade não foi acompanhada por medidas institucionais que efetivamente implementassem a política. Em conseqüência, as unidades de saúde vinculadas ao Estado não se sentem totalmente participantes do programa de saúde municipal.

A posição de intermediário nesse tipo de relacionamento é assumida muito fortemente pelo Hospital São Lucas da PUC, com índice betweeness de 207,5, e pelo Hospital Materno Infantil Presidente Vargas, onde esse índice alcança 55,5.

Se a coordenação municipal, por suposição, necessitasse estabelecer um programa de capacitação na identificação de pacientes e disseminar um protocolo para a referência aos serviços especializados, isso poderia ser feito através do Hospital São Lucas, o mais central e intermediário da rede, e do Hospital Nossa Senhora da Conceição, o segundo mais central.

A coordenação de caso ocorre quando um paciente é usuário de uma unidade de saúde e é encaminhado para algum serviço especializado para receber atendimento. Também ocorre entre CTAs e SAEs na adesão ao tratamento ou entre SAEs e hospitais no tratamento de pacientes. Esse tipo de relacionamento não é tão comum como os dois anteriores, pois implica maior grau de interação dos profissionais das unidades relacionadas. A densidade média foi de 7,5\%, contra 48,9\% no estudo de Kwait et al. (2001, p. 478). A centralidade desse tipo de relacionamento é exercida pelo SAE da Secretaria Municipal de Saúde, Ambulatório de Dermatologia Sanitária e SAE Partenon, coincidindo justamente os três SAEs da rede. A posição de intermediário nos relacionamentos de coordenação de caso é do SAE da Secretaria Municipal de Saúde, com índice de betweeness 223, seguido pelo Hospital de Clínicas de Porto Alegre, com 96, e pelo CTA/COAS Municipal, com 82.

A coordenação de caso, em determinadas situações, sucede em decorrência da existência de uma sub-rede, por exemplo do Centro de Saúde Murialdo. Em outras ocasiões, ela surge em função de relações pessoais entre profissionais, como nos relacionamentos do Ambulatório de Dermatologia Sanitária com os serviços de saúde comunitária do Grupo Conceição. A estruturação dos 
relacionamentos de caso não indica que haja uma prática efetiva e disseminada entre todas as unidades de saúde.

Os relacionamentos de programas conjuntos integram, principalmente, os programas de residência médica e de capacitação profissional. A densidade média foi de $4,5 \%$, contra $28,8 \%$ no estudo de Kwait et al. (2001, p. 480). A centralidade nesse tipo de relacionamento é do Ambulatório de Dermatologia Sanitária e do Hospital Nossa Senhora da Conceição. A posição de intermediário é assumida pelo CTA/COAS Partenon, com índice de betweeness de 96,5, e pelo Hospital Nossa Senhora da Conceição, com índice de 84. Não é por acaso que essas duas unidades de saúde figuram entre as de melhor posição intermediária, pois a primeira está ligada ao grupo de unidades do Centro de Saúde Murialdo e a segunda está ligada aos serviços de saúde comunitária do Grupo Conceição, ambos com sistemas próprios de assistência em saúde.

De maneira geral, os relacionamentos das unidades de saúde em função de programas conjuntos ocorrem porque estão situados num único ambiente organizacional, como é o caso do Centro de Saúde Murialdo e do Grupo Hospitalar Conceição, ou pelo conhecimento pessoal entre os profissionais, como é o caso da interação do Ambulatório de Dermatologia Sanitária com os serviços de saúde comunitária do Grupo Hospitalar Conceição. Não se observa a liderança desses programas por parte dos SAE's, que no desenho inicial do sistema figurariam como unidades de referência.

Através das entrevistas com os representantes das unidades de saúde não foi identificada a presença de relacionamento com base em consultorias, isto é, em serviços prestados a outras unidades da rede na base de contratos ou acordos formais. No estudo de referência de Kwait et al. (2001, p. 480), a densidade média foi de 5\%, num índice inferior aos anteriores daquele estudo, o que comprova a dificuldade de ocorrer este tipo de relacionamento.

\section{Estudo do Controle e Poder na Rede HIV/AIDS}

Compreender uma rede e seus relacionamentos é interessante para verificar a forma, a abrangência, a consistência e a dinâmica interna. Complementando esta análise, identifica-se qual das Unidades de Saúde exerce papel de liderança, a fim de se favorecer o processo de cooperação e integração que, em última análise, amplia as chances de alcançar melhores resultados aos pacientes.

A proposta é analisar três abordagens complementares da presença de poder e controle na rede: a densidade ou multiplexidade de serviço, o nível de centralidade da unidade de saúde principal e a concentração da influência na rede. Essas abordagens consideram a rede como um todo, isto é, todas as formas de relacionamento identificadas anteriormente agora são vistas conjuntamente.

A densidade de serviço é um escore do número de unidades de saúde conectadas por unidade especialista (Tabela 3). Assim, por exemplo, o Hospital Conceição conecta-se com 1,2 unidade da rede nos relacionamentos de envio, 7,5 nos relacionamentos de recebimentos, 1,1 nos relacionamento de coordenação de caso e 1,1 nos relacionamentos de programas, o que resulta num escore de 10,9 conexões por unidade de saúde da rede. O Hospital Conceição é a unidade de saúde especializada mais conectada com outras unidades, mas é seguida logo de perto pelo SAE da Secretaria Municipal de Saúde com 10,7 conexões. As duas unidades, desta forma, detêm o papel de centralidade entre as unidades de saúde especialistas e alcançam um escore superior ao dobro da média geral.

O nível de centralidade da unidade de saúde principal parte dos dados levantados no escore de densidade. Na teoria de redes sociais, a figura do líder facilita a coordenação do sistema, principalmente em redes de grande porte, como a formada pelos serviços de HIV/AIDS. Essa posição aparece com mais destaque, quando é ocupada por uma unidade que detenha mais da metade do escore da segunda. Por esse padrão, nenhuma das unidades de saúde especialista ocuparia a posição de unidade principal. 
Tabela 3: Densidade de Serviço na Rede de Unidades de Saúde Especializadas em HIV/AIDS

\begin{tabular}{|c|c|c|c|c|c|}
\hline Unidades & $\begin{array}{l}\text { Conexões de } \\
\text { envio }\end{array}$ & $\begin{array}{l}\text { Conexões de } \\
\text { recebimento }\end{array}$ & $\begin{array}{c}\text { Conexões } \\
\text { de coord. } \\
\text { de caso }\end{array}$ & $\begin{array}{l}\text { Conexões de } \\
\text { programas } \\
\text { conjuntos }\end{array}$ & $\begin{array}{l}\text { Densidade de } \\
\text { serviço }\end{array}$ \\
\hline $\begin{array}{l}\text { Hospital Nossa Senhora da } \\
\text { Conceição }\end{array}$ & 1,2 & 7,5 & 1,1 & 1,1 & 10,9 \\
\hline $\begin{array}{l}\text { SAE da Secretaria } \\
\text { Municinal de Saúde }\end{array}$ & & & & & \\
\hline \begin{tabular}{|l|} 
Municipal de Saúde \\
Hospital Vila Nova \\
\end{tabular} & $\begin{array}{l}3,5 \\
6,5 \\
\end{array}$ & $\begin{array}{l}4,6 \\
0,0 \\
\end{array}$ & $\begin{array}{l}2,5 \\
1,1 \\
\end{array}$ & $\begin{array}{l}0,1 \\
0,9 \\
\end{array}$ & $\begin{array}{r}10,7 \\
8,5 \\
\end{array}$ \\
\hline $\begin{array}{l}\text { Hospital São Lucas da } \\
\text { PUC }\end{array}$ & 0,0 & 7,9 & 0,0 & 0,0 & 7,9 \\
\hline $\begin{array}{l}\text { Hospital de Clínicas de } \\
\text { Porto Alegre }\end{array}$ & 0,8 & 3,4 & 0,9 & 0,2 & 5,3 \\
\hline \begin{tabular}{|l|} 
SAE Partenon \\
\end{tabular} & 2,1 & 0,0 & 1,5 & 0,8 & 4,4 \\
\hline \begin{tabular}{|l|} 
Ambulatório de \\
Dermatologia Sanitária \\
\end{tabular} & 1,4 & 0,0 & 1,5 & 1,4 & 4,2 \\
\hline $\begin{array}{l}\text { Hospital da Criança Santo } \\
\text { Antônio }\end{array}$ & 0,0 & 3,9 & 0,0 & 0,0 & 3,9 \\
\hline Hospital Fêmina & 0,0 & 3,3 & 0,0 & 0,0 & 3,3 \\
\hline \begin{tabular}{|l|} 
CTA/COAS Partenon \\
\end{tabular} & 0,9 & 0,0 & $\overline{0,6}$ & 0,9 & 2,5 \\
\hline \begin{tabular}{|l|} 
Irmandade Santa Casa de \\
Misericórdia de Porto \\
Alegre \\
\end{tabular} & 0,0 & 2,4 & 0,0 & 0,0 & 2,4 \\
\hline $\begin{array}{l}\text { Hospital Materno Infantil } \\
\text { Presidente Vargas } \\
\end{array}$ & 0,0 & 1,6 & 0,0 & 0,0 & 1,6 \\
\hline $\begin{array}{l}\text { Hospital Sanatório } \\
\text { Partenon }\end{array}$ & 1,0 & 0,0 & 0,0 & 0,2 & 1,2 \\
\hline CTA/COAS Estadual & 0,4 & 0,0 & 0,3 & 0,1 & 0,7 \\
\hline $\begin{array}{l}\text { CTA/COAS Municipal / } \\
\text { Paulo César Bonfim } \\
\end{array}$ & 0,3 & 0,0 & 0,1 & 0,1 & 0,5 \\
\hline Média & 1,2 & 2,3 & 0,6 & 0,4 & 4,5 \\
\hline $\mathrm{N}^{\circ}$ de Atores & 11 & 8 & 11 & 11 & \\
\hline
\end{tabular}

O grau de influência na rede foi obtido pelas respostas dos respondentes que identificaram, numa relação pré-definida de unidades de saúde especializadas, aquelas mais influentes nas decisões internas em relação ao atendimento dos pacientes com HIV/AIDS.

Pelas respostas oferecidas, a unidade de saúde mais influente é o SAE da Secretaria Municipal de Saúde, com $71 \%$ do escore máximo de influência. Esse escore não é mais do que o dobro do escore do CTA Municipal Paulo César Amorim, o segundo colocado. Seguindo o mesmo referencial de Provan e Milward (1995, p. 15), por esses dados, esse SAE não exerce uma influência concentrada na rede.

Durante o levantamento, verificou-se que os dados sobre influência apresentam limitações em razão da estruturação institucional do programa. As definições gerais de tratamento dos pacientes partem do Ministério da Saúde para o gestor local (município de Porto Alegre), que as repassa às unidades de saúde da rede. No futuro, estudos de rede social em sistemas de saúde deverão levar em conta esta influência na delimitação do objeto de análise.

Mesmo com tal limitação, é possível observar que as abordagens de análise de controle e poder na rede apontam o SAE da Secretaria Municipal de Saúde como detentor de posição de destaque na rede, embora sem sobressair. Por outro lado, a posição de líder pode não ser estática numa rede e se alterar em função da natural dinâmica dos relacionamentos.

A saúde em Porto Alegre é municipalizada e o programa de assistência em HIV/AIDS é de responsabilidade da Prefeitura de Porto Alegre. A vinculação e a subordinação funcional, além da 
afinidade organizacional do SAE com a Coordenação de DST do município, podem explicar essa posição assumida pela unidade, num recurso de governança da rede por meio da verticalização ou hierarquização (Grandori \& Soda, 1995).

\section{COMENTÁRIOS FINAIS}

A integração e a coordenação entre unidades da rede de saúde são altamente desejáveis num ambiente com pacientes crônicos e com alta especialização entre unidades. A simples definição de unidades com serviços especializados e a implementação de mecanismos de marcação de consultas com as demais unidades da rede de saúde não asseguram que haja uma efetiva interação entre elas. Neste estudo da rede de serviços de HIV/AIDS, os relacionamentos baseados em tais mecanismos, de envio e recebimento de pacientes, é que tiveram maior densidade nos relacionamentos. Entretanto, naqueles relacionamentos que necessitavam de uma iniciativa pela busca de colaboração e troca de informação, como na coordenação de caso, programas conjuntos e consultorias, os índices de densidade foram bem inferiores; não foi identificada a existência de consultorias. Além disso, muitas iniciativas, nestes últimos tipos de relacionamento, estavam baseadas em práticas de algumas unidades ou em relações pessoais, ao invés de considerar primeiro a necessidade assistencial do pacientes para direcionar, a partir daí, os relacionamentos, como defendido por Kwait et al. (2001).

A compreensão da dinâmica de uma rede, como a de HIV/AIDS, pode acelerar a disseminação de novos conhecimentos, o diagnóstico de falhas na implementação da política de saúde e a otimização da estrutura organizacional, como a identificação, no presente estudo, da divisão da liderança entre os hospitais e os serviços de assistência especializada [SAE].

Há necessidade de aprimorar a governança, por exemplo com o acompanhamento do recebimento de pacientes, coordenação de casos entre unidades e realização de programas conjuntos. Um dos princípios do SUS é a estruturação hierárquica, o que implica a adoção de vínculos entre unidades de saúde primárias, secundárias e terciárias. O Grupo Conceição e o Centro de Saúde Murialdo criaram, por iniciativa própria, uma rede de serviços com essa estrutura, mas isso não é praticado pelo restante da rede. No estudo de Kwait et al. (2001), havia uma política explicita de integração entre as unidades de saúde, reconhecida como fator indutor no sistema local.

A literatura de saúde é enfática na defesa da integração dos serviços para a obtenção de melhores resultados assistenciais, principalmente no atendimento das necessidades dos pacientes; esta diretriz foi incorporada no projeto institucional do Sistema Único de Saúde brasileiro. O perfil epidemiológico das doenças tem mudado com o crescimento de doenças crônicas como o HIV/AIDS, que consomem maior quantidade de recursos dos sistemas. Neste contexto, a desejada integração se justifica e tornase ainda mais necessária (Kitahata et al., 2002). No estudo de Wright e Shuff (1995), a busca de integração entre serviços de atendimento mental e de HIV/AIDS levou à ampliação da cooperação com outros serviços da rede.

Ainda assim, pouco se conhece a respeito das interfaces interorganizacionais para se ter uma avaliação da qualidade de tais relacionamentos. Observa-se que o termo rede muitas vezes não passa de uma denominação para um conjunto de unidades de saúde, que não realizam uma efetiva integração.

Como se pode verificar, a partir deste estudo de caso, há inúmeras características a serem observadas nas transações, como a governança, a estrutura e a dinâmica da rede. Ainda que com certas limitações, verificou-se que os sistemas estudados funcionam como redes de serviços muito tênues, sem evidentes ações de parceria e sem marcantes ações de governança. 
O estudo de redes sociais permite a leitura de grande número de organizações, sendo operacionalmente rápido de executar, possibilitando a identificação dos atores mais importantes, dos laços mais fortes entre eles e, também, da ausência ou fraqueza de relacionamentos.

Partindo-se das prioridades do sistema e de um acompanhamento das redes sociais, cada organização individualmente pode rever sua forma de interagir com outra unidade de saúde, e o gestor do sistema tem a oportunidade para replanejar o desenho, funcionalidades e prioridades do sistema.

Desta forma, acredita-se que o objetivo principal deste trabalho tenha sido atingido, isto é, demonstrar as potenciais contribuições da análise de redes sociais em saúde.

O aprimoramento e a consolidação dessa linha de pesquisa abrem caminho para estudos em redes de outros tratamentos de saúde, para a utilização de dados longitudinais de redes interorganizacionais de saúde e para a análise de redes sociais dentro de etapas de evolução da doença.

\section{Artigo recebido em 13.08.2007. Aprovado em 18.08.2008.}

\section{REFERÊNCIAS BIBLIOGRÁFICAS}

Borgatti, S. P., Everett, M. G., \& Freeman, L. C. (2002). Ucinet for windows: software for social network analysis [software]. Harvard, MA: Analytic Technologies.

Borgatti, S. P., \& Foster, P. C. (2003). The network paradigm in organizational research: a review and typology. Journal of Management, 29(6), 991-1013.

Breiger, R. L. (1974). The duality of person and groups. Social Forces, 53(2), 181-190.

Conrad, D. A., \& Shortell, S. M. (1996). Integrated health systems: promise and performance. Frontiers of Health Services Management, 13(1), 3-40.

Delnoij, D. M. J., Klazinga, N. S., \& Velden, K. van der. (2003). Building integrated health systems in central and eastern Europe. European Journal of Public Health, 13(3), 240-245.

Draibe, S. M. (1999). As políticas sociais nos anos 90. In R. Baumann (Org.). Brasil: uma década em transição (pp. 101-142). Rio de Janeiro: Campus.

Feld, S., \& Elmore, R. (1982). Patterns of sociometric choices: transitivity reconsidered. Social Psychology Quarterly, 45(2), 77-85.

Fontana, A., \& Frey, J. (2000). The Interview. In N. K. Denzin, \& Y. S. Lincoln (Eds.). Handbook of Qualitative Research (2nd ed., pp. 645-672). California: Sage.

Freeman, L. C. (1996). Some antecedents of social network analysis. Connections, 19(1), 39-42.

Friedman, L., \& Goes, J. (2001). Why integrated health networks have failed. Frontiers of Health Services Management, 17(4), 3-28.

Grandori, A., \& Soda, G. (1995). Inter-firm networks: antecedents, mechanisms and forms. Organizations Studies, 16(2), 183-214.

Grandori, A., \& Soda. G. (2000). A relational approach to organization design [Working Paper]. Center for Research on Organization and Management, Milan: Bocconi University. 
Hanneman, R. A., \& Riddle, M. (2005). Introduction to social network methods. Riverside, CA: University of California. Recuperado em 23 novembro, 2004, de http://faculty.ucr.edu/ hanneman/

Institute of Medicine (2001). Crossing the quality chasm, a new health system for the 21st Century. Washington: National Academy Press.

Kadushin, C. (2002). The motivational foundation of social networks. Social Networks, 24(1), 77-91.

Kitahata, M. M., Tegger, M. K., Wagner, E. H., \& Holmes, K. K. (2002). Comprehensive health care for people infected with HIV in developing countries. British Medical Journal, 325(7370), 954957.

Kwait, J., Valente, T. W., \& Celentano, D. D. (2001). Interorganizational relationships among HIV/AIDS service organizations in baltimore: a network analysis. Journal of Urban Health: Bulletin of the New York Academy of Medicine, 78(3), 468-487.

Light, D. W. (2001). Managed competition, governmentality and institutional response in the United Kingdon. Social Science \& Medicine, 52(8), 1167-1181.

Luke, D. A., \& Harris, J. K. (2007). Network analysis in public health: history, methods, and applications. Annual Review of Public Health, 28, 69-93.

Marques, E. C. (2000). Estado e redes sociais - permeabilidade e coesão nas políticas urbanas no Rio de Janeiro. São Paulo: Revan.

Marsden, P. (1987). Discussion networks of Americans. American Sociological Review, 52(1), 122131.

Martes, A. C. B., Loureiro, M. R., Abramovay, R., Serva, M., \& Serafim, M. (2006). Fórum Sociologia Econômica. Revista de Administração de Empresas, 46(3), 10-15.

Milward, H. B., \& Provan, K. G. (1998). Measuring network structure. Public Administration, 76(2), 387-407.

Ministério da Saúde. (2007). Cadernos de informação de saúde. Recuperado em 8 abril, 2008, de http://tabnet.datasus.gov.br/tabdata/cadernos/cadernosmap.htm

Mizruchi, M. (1994). Social network analysis: recent achievements and current controversies. Acta Sociologica, 37(4), 329-343.

Moe, T. L. (2005). Managerial networking of public and private sector: a study of thai executives. Public Administration and Management, 10(4), 278-324.

Monnerat, G. L., Senna, M. C. M., \& Souza, R. G. (2002). A reorganização dos serviços de saúde no cenário local. Ciência \& Saúde Coletiva, 7(3), 509-521.

Oliver, A. L., \& Ebers, M. (1998). Networking network studies: an analysis of conceptual configurations in the study of inter-organizational relationships. Organization Studies, 19(4), 549-583.

Owen-Smith, J., \& Powell, W. (2004). Knowledge networks as channels and conduits: the effects over spillovers in the Boston biotechnologies community. Organization Science, 15(1), 5-21.

Provan, K. G., \& Milward, H. B. (1995). A preliminary theory of interorganizational network effectiveness: a comparative study of four community mental health systems. Administrative Science Quarterly, 40(1), 1-33. 
Rank, C., Rank, O., \& Wald, A. (2006). Integrated versus core-periphery in regional biotechnology. European Management Journal, 24(1), 73-85.

Secretaria Municipal de Saúde de Porto Alegre. (2007). Boletim epidemiológico [Ano IX, No 34]. $\begin{array}{llll}\text { Recuperado em } & 8 & \text { abril, }\end{array}$ http://lproweb.procempa.com.br/pmpa/prefpoa/sms/usu_doc/boletim_n_34_novo.pdf

Stiles, R. A., Mick, S. S., \& Wise, C. G. (2001). The logic of transaction cost economics in health care organization theory. Health Care Manage Review, 26(2), 85-92.

The Institute for the Future. (2003). Health and health care 2010: the forecast, the challenge (2nd ed.). Princeton, NJ: Princeton University.

Tichy, N., Tushman, M., \& Fombrun, C. (1979). Social network analysis for organizations. The Academy of Management Review, 4(4), 507-519.

Wasserman, S., \& Faust, K. (1994). Social network analysis, methods and applications. Cambridge, UK: Cambridge University Press.

Wright, E. R., \& Shuff, I. M. (1995). Specifying the integration of mental health and primary health care services for persons with HIV AIDS: the Indiana integration of care project. Social Network, 17(3-4), 319-340. 